\title{
DU BON USAGE DES SOURCES EN HISTOIRE CULTURELLE. ANALYSE COMPARÉE DES PROCÈS D’INQUISITION ET DES PROCÈS DE BÉATIFICATION
}

\author{
Jean-Michel Sallmann \\ Université Paris X-Nanterre \\ Pesquisador do Centre d'Études et Recherches sur les mondes américains - EHESS
}

RESUMO: Este artigo preocupa-se em refletir sobre o problema das fontes históricas e do crédito que nelas podemos depositar. Ele poderia exprimir-se numa simples pergunta: $\mathrm{em}$ que medida a percepção que temos de um fenômeno é tributária da fonte que utilizamos? Tomemos o exemplo dos arquivos judiciârios: a leitura de seus documentos revela características fortemente alteradas pela intervenção do juiz, a transcriçāo do escrivão etc. Para isto, estudarei, em paralelo, os processos de beatificação e da inquisição no sul da Itália na época moderna.

ABSTRACT: This article discusses the issue of historical sources and their credibility. This can be addressed in a simple question: in what way is our perception of a phenomenon based on the source we use? Let us take as an example the judiciary archives: the reading of its documents reveal characteristics altered by the intervention of the judge, the clerck's transcription etc. To further illustrate the subject, the beatification and inquisition documents in southern Italy during the Modern Age are studied.

PALAVRAS-CHAVE: História Cultural, Crítica Documental, Inquisição, Bcatificaçāo, Processos.

KEY-WORDS: Cultural History, Documental Criticism, Inquisition, Beatification, Documents.

Cet articlc voudrait réfléchir sur un problème qui se trouve à la base de notre activité d'historien, celui des sources et du crédit qu'on peut leur accorder. II pourrait s'exprimer dans une simple question: dans quelle mesure la perception que nous avons d'un phénomène est-elle tributaire de la source que nous utilisons? Dire qu'une source n'est jamais neutre relève du poncif historiographique, mais il semble bon de lc rappeler de temps à autre. L'archive est le produit d'une administration. Elle est rédigée selon une logique qui est propre à l'administration qui l'a émise mais qui ne correspond pas toujours à ce que l'historien attend d'elle. Certains exemples constituent des cas d'école. Un texte normatif - une ordonnance, un édit, une cédule, une pragmatique - représente un ideal rarement atteint. La multiplication de ces mêmes textes sur le long tèrme témoigne de leur inefficacité ou du moins des difficultés à faire entrer leur application dans les faits. Un historien en histoire sociale sait aussi qu'il 
doit se méfier des documents d'origine fiscale entachés de fraude.

Il en va de même en histoire culturelle. Je voudrais prendre l'exemple des archives judiciaires dont les historiens rcconnaissent généralcment qu'elles livrent des témoignages bruts. La pratique assidue de ces documents montre pourtant que leur caractère spontané est fortement altéré par les contraintes de la procédure, les interventions du juge, la transcription du greffier. Loin de moi de prétendre que les archives judiciaires n'ont aucune valcur, mais il est nécessaire de comprendrc dans quel état d'esprit et à quelle fin elles ont étć produites pour en tirer le profit maximum... et éviter les contre-sens. Pour cela, j'étudierai en parallèle les procès de béatification et les procès d'inquisition dans le domaine que je connais le moins mal, I'Italic du sud a l'époque moderne.

\section{Deux procédures parallèles et antinomiques}

Il existe entre ces deux types de documents beaucoup plus de points de contact qu'on ne le soupçonnc généralement. lls sont nés au même moment, vers 1200 , et sont fondés tous les deux sur la même procédure, la procédure inquisitoire. Cette histoire commune se poursuit pendant longtemps, puisqu'ils atteignent de manière simultanée - à la fin du XVI et au début du XVII e siècle - leur forme la plus élaborée. Avant d'aller plus loin dans cette étude, il convient de préciser un certain nombre de notions indispensables. En matière criminelle, les codes de lois du haut Moyen-Age, inspirés des traditions germaniques, reposaient sur la procédure accusatoire. Un procès n'étaìt ouvert qu'à partir du moment où le plaignant portait une accusation contre son agresseur devant le tribunal. Ce tribunal constitué d'experts (boni homines, prudentes homines) était chargé de déterminer si l'accusation était fondée et, au cas où elle l'était, de fixer le montant de la compensation financière à verser à la victime pour le tort subi. Mais si, dans le cadre du débat contradictoire qui opposait l'accusateur et l'accusé, ce dernier parvenait à démontrer son innocence, l'accusateur était condamné à là peine qu'aurait subie l'accusé s'il avait été rcconnu coupable. La procédure inquisitoriale, qui s'impose dans le droit canonique aux environs de 1200 por gagner ensuite le droit pénal, repose sur d'autres principes. Un juge procède à une enquête - d'où le nom de la procédure - sur un délit dont il a eu connaissance. Il instruit l'affaire en interrogeant le suspect et les témoins, se fait une conviction sur la base des renseignements ainsi recueillis, au besoin par la torture, et émet la sentence.

\subsection{Les procès de béatification}

La procédurc en béatification est récente puisqu'elle ne date que du début du XVII siècle, mais elle s'inspire de la procédure en canonisation qui s'cst mise en place au XIIl' siècle, lors de la grande révolution juridique qui bouleverse le droit canonique à cette époque.

A l'époque médiévale, les deux procédures de béatification et de canonisation cocxistent mais sont très différentes. La canonisation consiste ì déclarer saint un serviteur de Dieu mort en réputation de sainteté, et donc à lui accorder un culte universel. Elle est du ressort exclusif de la papauté et une décrétale du pape Alexandre III dans les premières années du $X I I I^{e}$ siècle institue ce droit de réserve pontificale. A la suite d'un procès en bonne et due forme qui analyse successivement les écrits, la vie et les miracles du serviteur de Dieu, le pape inscrit officiellement le personnage au catalogue des saints. La béatification, au contrairc, relève des églises locales. Le clergé d'un diocèse accorde le titre de bienheureux (beatus) à un serviteur de Dieu, que l'évêque élève sur les autels. Pratiquement, les reliques du nouveau bienheureux étaient enfermées 
dans une châsse et placées sur un autel pour recevoir un culte strictement local (VAUCHEZ, 1981).

Mais tout change à la fin du XVIe siècle à la suite de deux innovations importantes. En 1588 est créée la Congrégation des Rites, congrégation de cardinaux chargée de contrôler les formes du culte, en particulier du culte du aux saints. Très rapidement, cet organisme reçoit la tâche d'instruire les procès de canonisation qui jusque-là, étaient du ressort du tribunal de la Rotc. D'autre part, avec l'impuision donnée à la Contrc-Réforme, les cultes spontanés se multiplient envers les grandes figures du catholicisme du temps, comme Filippo Neri et Ignace de Loyola à Rome ou Carlo Borromeo à Milan. Pour contrôler celte explosion anarchique, le Saint-Siège éprouve le besoin d'imposer son droit de réserve, qui devient officiel par deux décrets d'Urbain VIII en 1625 et 1634, mais qui est déjà réel des la fin des années 1610. Même si le bienheureux ne bćnéficie que d'un culte local, le procès de béatification est construit sur le modèle du procès de canonisation. La béatification devient ainsi une première étape sur la voic de la canonisation (SALLMANN, 1994). L'historicn de l'époque moderne, sensible à l'aspect social et culturel du culte des saints, utilisera plus volontiers les procès de béatification, dont les témoignages lui sembleront plus riches que les informations stćrćotypćcs contcnucs dans les procès dc canonisation.

Deux principes président à la réalisation d'un procès en béatification: la minutie et le temps, considérés comme le gage d'un bon choix chez ceux qui sont destinés à prendre la décision, les cardinaux de la Congrégation des Rites et le pape, en dernier ressort. C'est pourquoi cette procédure cst lente et redondante. Elle commence par un procès informatif, qui est une enquĉte informelle, au cours de laquelle le postulateur de la cause recuielle tous les témoignages concernant le serviteur de Dieu, ses écrits, ses éléments biographiques et surtout ses miracles, généralement enregistrés devant notaire. Cette partie de la procédure a souvent disparu, les archives diocésaines n'en conservant que des épaves dans le meilleus des cas.

La deuxième étape est représentée par le procès diocésain, ouvert dès que possible sous la présidence de l'évêque du lieu et dont la cheville ouvrière est une nouvelle fois le postulateur. Ce dernier rédige les positions - c'est-à-dire l'ensemble des éléments biographiques et légendaires qu'il a pu recueillir -, les questionnaires sur lesquels les témoins sont interrogés. Le procès diocésain constitue ainsi la dernière trace de l'initiative locale qui prévalait avant que le Saint Siège n'impose son droit de réserve. Une copie de ce procès est envoyée à la Congrégation des Rites qui nomme un cardinal appelé cardinal-ponent - chargé du suivi de l'affaire. S'il obtient la signature du pape, la cause est introduite en congrégation. S'ouvre alors le procès apostolique, réalisé sous l'autorité de la congrégation. Le promoteur de la foi - le fameux avocat du diable - nomme deux commissaires, des évêques généralement, chargés d'instruire des procès dans tous les lieux où le serviteur de Dieu a laissé des souvenirs. Une copie authentique de ces procès est exécutée, envoyée à la Congrégation, misc sous sceltés et classée dans ses archives où elle doit dormir théoriquement pendant 50 ans. C'est alors sculement que le procès est à nouveau ouvert. De longues discussions animées par le postulateur qui défend la cause et par le promoteur qui en vérifie la régularité aboutissent à une série de votes, sur les vertus puis sur les miracles. Si les votes sont positifs, le pape publie le décret de béatification.

\section{2. Les procès d'inquisition}

A priori, nous nous retrouvons dans un domaine différent, puisque l'Inquisition a pour fonction de réprimer l'hérésie. A y regarder de plus près pourtant, les choses sont plus compliquées et les points de 
contact entre la procédure inquisitoriale et la procédure en béatification sont nombreux. L'institution inquisitoriale naît au tut début du XIlle siècle ct connaît un grand développement au moment de la répression contrc les mouvements cathare ct spiritucl. Après une éclipse à la fin du Moyen-Age liée à la crise de l'Eglise et à la montée en puissance des États territoriaux qui supportent mal l'existence d'un tribunal directement affilić à la Papauté, I'Inquisition revit au $\mathrm{XVI}^{\mathrm{e}}$ siècle. L'Inquisition espagnole est reformée en 1478-1480, puis c'est au tour de l'Inquisition romaine de connaître un regain à partir de 1542.

Il ne faut pas oublier qu'au XVIe siècle la Congrégation du Saint-Office a servi de modèle aux autres Congrégations romains, comme la Congrégation des Rites, et que l'activité de ces deux Congrégations a été perçue comme complémentairc, l'une réprimant l'hérésie protestante, et l'autre promouvant ct défendant les formes de culte spécifiques au catholicisme. Les décrets par lesquels Urbain VIII instaure en 1625 et 1634 le droit de réserve pontificale en matière de béatification sortent des bureaux de la Congrégation de Saint-Office et non pas de ceux de la Congrégation des Rites. Leur destination est purement répressive puisqu'ils visent à interdirc des formes dévoyées du culte rendu à des serviteurs de Dieu non encore béatifiês.

La procédure inquisitoriale présente des affinités avec la procédure en béatification, surtout dans les régions dépendant de l'Inquisition romaine comme à Naples. C'est le tribunal diocésain qui enquête et qui juge en matière de foi sur comission du Saint-Office, et le représentant de la Congrégation est soit l'êvêque dans les diocèses de faible étendue, soit lc vicaire général dans les gros diocèses comme celui de Naples. Quand les circonstances l'exigent, que la lutte contre l'hérésie se fait plus pressante ou que le ressort du diocèse est trop étendu pour le seul vicairc général, la Congrégation romaine nomme un ministre du Saint-Office qui ne forme pas autour de lui un tribunal permanent, mais qui est chargé d'aider le tribunal diocésain en instruisant certaines affaires en matière de foi.

Le procès inquisitorial commence lui aussi par une enquête informelle chargée de recueillir les dénonciations, les dépositions des témoins à chargé, les interrogatoires des inculpés, dont les éléments sont envoyés à la Congrégation romaine du SaintOffice. Sauf dans les cas très graves qui nécessitent le transfert des accusés sous escorte armée à Rome, les procès sont assurés localement, soit de manière expéditive, soit dans le respect des formes canoniques. Dans ce dernier cas, l'inculpé peut présenter sa défense. Mais, en tout état de cause, la Congrégation romaine du Saint-Office évoque toujours les dossiers en matière de foi, conseille le ministre local du Saint-Office ou le vicaire général, suggère les peines à infliger (SALLMANN, 1986).

A ce point de la comparaison, il est possible de dresser un premier bilan. L'étroite parenté entre la procédure inquisitoriale et la procédurc en béatification permet de définir des caractères communs et des divergences. Parmi les caractères communs, notons le rôle joué par les deux congrégations romaines de cardinaux, qui exercent un contrôle étroit tout au long de la procédurc. Il est fréquent d'ailleurs que le personnel passe de l'une à l'autre en cours de carrière. D'autre part, les procès sont instruits et jugés par des juges commissaires en collaboration étroite avec les juges ordinaires qui peuvent recevoir à l'occasion une commision spéciale.

On peut relever, par contre, des divcrgences sur plusicurs points. Dans la procédure en béatification, les juges locaux sont dessaisis de toute initiative. Dans la premièrc phase d'instruction, ils se contentent de collecter les informations. Le travail judiciaire proprement dit, cclui qui aboutit à l'émission de la sentence, revient à la Congrégation 
qui vote, favorablement ou non. La décision finale au pape. A l'inverse, la procédure d'inquisition est locale. Même si la Congrégation du Saint-Office surveille et conseille le tribunal diocésain, c'est ce dernier qui vote la sentence, et parfois contre l'avis de la Congrégation romaine. D'autre part, si la procédure en béatification est lente et s'etend sur plusieurs décenniers, la procédure inquisitoriale est dictée par l'urgence.

Mais, tout compte fait, ces différences sont formelles et ne changent pas substantiellement la nature de la parenté qui lie ces deux procédures. Elles déterminent plutôt deux états d'esprit et masquent avec difficulté des a priori ideologiques. A l'ouverture d'un procès en béatification ou d'un procès d'inquisition, les jeux sont déjà quasiment faits et le reste n'est qu'anecdotique. Le serviteur de Dieu est dćjà considéré comme un saint avant même l'ouverture de son procès et reçoit un culte discret ou des honneurs plus tapageurs. Au-delà des aléas dc la procédure, il ne faut pas oublier qu'une cause en béatification n'est jamais terminée. Tant qu'elle n'a pas abouti, elle est mise cn sommeil mais peut être réactivée à tout moment. De la même maniére, un individu inculpé devant un tribunal inquisitorial est déjà sinon condamné d'avance, du moins fortement soupçonné d'hérésie ou de déviance religieuse. On ne sort jamais indemne d'un procès devant I'Inquisition. Ce sont donc ces deux états d'esprit que je vais m'efforcer d'analyser.

\section{L'image de la vraie sainteté}

\subsection{De l'enquête biographique à la légende}

Un procès de béatification doit établir, à travers le plus grand nombre de témoignages possible, la sainteté d'un serviteur de Dieu ou d'une servante du Christ selon la terminologie utilisée. L'enquête biographique est réalisée par le postulateur qui rédige les positions sur la base desquelles le promoteur de la foi dresse les interrogatoires. Les juges convoquent les témoins en sélectionnant les chapitres des positions et les questions auxquels ccux-ci sont susceptibles de rêpondre.

Pourtant, les actes des procès et la littérature hagiographique qui en est directement inspirée renvoient une image stéréotypée du futur saint, sélectionnéc à partir de quelques anecdotes auxquelles on peut accorder quelque crédit. On y retrouve dans l'ordre: une naissance merveilleuse, une enfance pieuse, une entrée au couvent suivant une crise familiale ou personnelle, une conversion à la vie sainte plus ou moins tardive, une exigence de rigueur, une mise en apprentissage auprès d'un maître spirituel. A partir de ce moment, le futur saint mène une vie exemplaire: il exerce les vertus chrétiennes au degré heroique, s'administre des jeûnes et des macérations, accomplit des miracles, puis meurt d'une belle mort chrétienne au milicu d'un grand concours de peuple, les prodiges et les miracles se multipliant après sa mort. Le tout est agrémenté de tous les lieux communs de la tradition hagiographique.

Les raisons de ce caractère stéréotypé tiennent aux circonstances qui entourcnt l'ćlaboration du procès. Tout d'abord, pour la plupart des témoins, le futur saint est quasi un inconnu. Très peu l'ont connu de son vivant: quelques religieux dans les couvents où il est passé, quelques disciples laiques qui, en vertu de leur position sociale, ont eu la chance de s'entretenir avec lui dans sa cellule, quelques tertiaires dont il etait le directeur de conscience. Pour les femmes, l'obligation de clôture restreint encore les possibilites de contact avec le monde extérieur. Il faut ajouter que, dans une enquête en béatification, la partie du procès qui a le plus grand poids est celle qui se déroule dans le lieu où est mort le saint. Ce lieu n'est pas forcément celui où il a passé le plus clair de son existence. Certains 
saints, comme Bernardino Realino à Lecce ou Andrea Avellino à Naples, tous deux morts au début du XVII ${ }^{c}$ siècle, se sont révélés très casaniers et sont restés dans le mème couvent, mais la plupart ont fréquemment changé de résidence, ce qui contribue à atténucr leur "mémoire vive".

Par ailleurs, la saintcté est une qualité inéluctable à laquelle le futur saint ne peut guère échapper. On est saint, on le devient pas. La conversion à la sainteté est le résultat d'un appel de Dicu, soudain et irrépressible. Dès qu'il l'a recu, le futur saint change totalement de comportement. Quelque chose en lui respirc la sainteté: il en porte les signes distinctifs (des habits en loques, un chapeau d'une coulcur inhabituclle, les cheveux ras) et commence à ressentir des états spécifiques (extases, lévitation, chorée publique). S'établit alors une complicité entre le saint et ses fidèles. Le promier revendique officiellement son statut de sainteté, mais, à l'inverse, le public le perçoit comme tel et tous ses gestes sont interprétés cn fonction de cette seule conviction qu'il est un saint. Le procès de béatification est justement chargé de recueillir cette conviction générale. Sa fonction consistc à dés-historiciscr les ćléments biographiques du personnage pour les ajuster au micux à quelques prototypes bien ćprouvés. Le meilleur exemple en cst le don de prophétic, attribut obligatoire de toute sainteté. Le saint est consé prophétiser sa mort prochaine. L'analyse des dépositions montre que l'interprétation se fait toujours a posteriori. Après la mort, le témoin se souvient que le saint luj avait tenu des propos qui, à l'époque, lui avaient paru obscurs, mais par lesqucls, tout bien pesé, il lui a semblé qu'il annonçait sa mort prochaine.

Toutcs ces conditions aboutissent à la constitution d'un légendaire qui contribue à faire glisser la vie du saint d'une biographie à une hagiographie. Bien souvent, il sc moule sur une tradition bien établie. C'est le cas, par exemple, chez les Franciscains qui, à l'imitation de saint François, doivent parler aux oiseaux et calmer les betes enragées. Ce légendaire fait aussi l'objet d'une appropriation de la part des groupes familiaux ou politiques qui prennent en chargé la cause en béatification. Nous en avons un exemple à la fin du $X V I^{c}$ siècle avec un franciscain d'Amalfi, Domenico da Muro. Au fur et à mesure que le procès progresse, sa vie se résume à deux épisodes prodigieux, lá resurrection du capitaine de la tour de guet d'Amalfi et le sauvetage miraculeux d'une frégate maltaise dans la baic d'Amalfi. Ces deux miracles sont utilisés par deux familles roturic̀res puissantes de la région, les Anastasio ct les Salati. Ils correspondent à deux temps dans l'enquête, deux procès de béatification successifs, contrôlés par chacune de ces familles.

\subsection{Miracles et groupes de pression}

Il serait donc vain d'aller chercher dans un procès de béatification ce qu'on ne peut $y$ trouver. Non pas que ce qu'on y trouve n'a pas d'intérêt, mais il fournira des informations sur les représentations symboliques de la sainteté et non sur la vie du saint. Une fois cette leçon bien assimiléc, if est facile de comprendre en quoi consistc exactement un procès de béatification. C'est un catalogue de miracles. Nous avons vu que dans la phase informative, le postulateur de la cause se chargeait d'en recueillir le plus grand nombrc. Dans les procès diocésains et apostoliques, les miracles sont situés en fin des positions et la plupart des témoins ne sont interrogés que sur ces derniers articles. Au XVII ${ }^{e}$ siècle, le miracle est encorc considéré comme le signe surnaturel de l'élection divine, la preuve la plus éminents de la sainteté du personnage.

On éprouve aujourd'hui des difficultés à imaginer la fréquence du miracle dans la vie quotidienne, mais les chiffres sont là sans contestation possible. Entre 1613 et 1619 ont été 
relevés sur le tombeau du théatin de Naples, Andrea Avellino, 2158 ex-voto alors qu'il étair mort en 1608 et qu'il ne fut béatifié qu'en 1624. Les relevés opérés dans quelques procès de béatification meridionaux donnent les resultats suivants: pour Andrea Avellino toujours 8 miracles de son vivant et 125 après la mort; pour Bernardino Realino à Naples et à Lecce 170 miracles de son vivant et 220 après la mort; pour Domenico da Muro à Amalfi 30 et 30 ; enfin pour Bonaventura da Potenza au début du XVIII ${ }^{\mathrm{e}}$ siècle dans quelques sites de la péninsule de Sorrente 20 et 47.

Plusieurs conclusions s'imposent. Les miracles sont déterminants dans une cause en bêatification. Ils en constituent l'ultima étape, quand l'heroicité des vertus a été reconnue par la Congrégation. La reconnaissance de l'authenticité des miracles induit automatiquement la béatification. II n'existe par ailleurs aucun rapport théorique entre le nombre des miracles rclevés par les juges et l'accès aux autels. Les textes canoniques prévoient que 2 miracles authentiques -4 au XVIII ${ }^{\mathrm{e}}$ siècle - suffisent. II n'empêche. Parmi les exemples cités plus haut, il convient dc remarquer qu'Andrea Avellino et Bernardino Realino ont été canonisés, que Bonaventura da potenza a été béatifié, mais que la cause de Domenico da Muro s'est arrêtée au stade de la reconnaissance de ses vertus.

Pour constituer le procès en béatification, les juges et lc postulateur opèrent donc un tri. De ce point de vue, la différence entre le nombre d'ex-votes déposés sur le tombeau d'Andrea Avellino et le nombre des miracles recueillis dans son procès est révélatrice. Elle est due à plusieurs raisons: beaucoup de ces dons restent anonymes et disparaissent avec le temps au fond d'une sacristie, surtout après le décret pontifical de 1625 . D'autre part, les juges ćtablissent leur sélection selon des critères sociaux. Sur plus de 1000 témoins relevés dans les procès du $\mathrm{XVII}$ siècle, $18 \%$ seulement proviennent des classes populaires urbaines, pour ainsi dire aucun de la paysanneric. Tout le reste est issu de l'aristocratic principalement, du clergé et, dans une moindre mesure, de la bourgeoisie. Il n'est oas étonant de voir Benoît XIV au XVIII' siècle rappeler qu'il est préférable de choisir les témoins parmi les gens honorables, riches ct cultivés. Autrement dit, un témoignage est d'autant plus légitime, un miracle d'autant plus authentique qu'ils émanent du sommet de la société.

\subsection{Vox populi, vox Dei}

On peut résumer ainsi les fonctions principales d'un procès en béatification. Il s'agit tout d'abord de gommer tout trait de la personnalité du prétendant à la sainteté, ou plutôt de ramener les épisodes de sacie à des stéreotypes qui s'accorderaient au mieux avec une mythologie hagiographique. Les anthropologues parlent à cet effet d'un procèssus de dés-historicisation, puis d'intégration dans un modèle déjà éprouve (MARTINO, 1972; 1975; 1976). Le succès remporté par un saint vient de ce qu'il réussit à se conformer à l'image que son public attend de lui. Il s'y emploie dès son vivant, mais le procès et le travail de réćlaboration qui láccompagne achèvent cette identification. C'est alors que le saint peut entrer dans une cour céleste nombreuse et indifférenciéc.

D'autre part, le procès sert à établir le degré atteint par la renommée de sainteté, la fama sanctitatis. Le procès ne la crée pas, car elle existe du vivant du saint. Il en garde la mémoire et il l'amplifie. Grâce au procès, le groupe de dévots qui soutient la cause, pour des raisons de dévotion et de prestige, peut se compter et se mobiliser. C'est pourquoi la vox populi est toujours socialement sélective. La procédure est longue et hasardeuse et la sélection s'opère par l'échec. Il faut de l'argent et de l'entregent pour entretenir les postulateurs et les procureurs chargés de vaincre les obstacles 
bureaucratiques. Sculs les groupes de pression puissants et organisés sont capables de franchir tous les aléas de la procédure.

Si un serviteur de Dieu n'a personne pour le soutenir, sa renommée de sainteté s'effondre d'ellemême en peu de temps. On possède des traces de renommée tronquée avec ces ébauches de procès qu'on rencontre fréquemment dans les archives diocésaincs ou conventuelles. Par contre, si le groupe de pression parvient à enclencher la procédure, celle-ci démultiplie ses effets sur la fama. Chaque étape donne l'occasion de remobiliser les partisans de la cause, de réaliser quelque nouveau ritucl (ouvrir le tombeau par excmple), de publier une nouvelle hagiographie, ce qui finit toujours par générer de nouvcaux miracles, de nouveaux prodiges. Dans ce contexte, le décret de béatification intervient pour ainsi dirc naturellement, commc unc réponse à unc demande universelle. On voit done que la procédure produit par ellc-même des effets qui ne sont pas prévus par le code canonique. Ces mêmes effets, on les retrouve dans la procédure inquisitoriale, mais inversés.

\section{L'image de la fausse sainteté}

\subsection{L'Inquisition ou la mauvaise réputation}

La réputation de sainteté constitue done la colonne vertébrale d'un procès en béatification, partagée de la même manic̀re par l'obscur serviteur de Dieu et par le grand saint, au point qu'il est impossible de déterminer à partir de critères objectifs pourquoi cehui-ci a été retenu plutôt que celui-là. A l'époque moderne, l'lalie du sud n'a connu qu'un seul cas de refus clairement exprimé par la Congrégation des Rites pour deux raisons précises: cet ermite - Carlo de Mercantiis - se vantait faussement de posséder des ascendances nobillaires et offrait du chocolat à ses visiteurs. Pour le reste, comme aucune cause ouverte n'est jamais définitivement close, on doit considérer que tout procès qui n'a pas officiellement abouti peut être repris et mené à bien à tout moment.

A l'inverse, lcs procès d'inquisition se fondent sur la réputation d'hérésie. Il faut imaginer ce qu'cst le travail d'un tribunal d'inquisition dans une Italie méridionale qui ne fut jamais menacée par le protestantisme $c$ où la faiblesse de la présence juive et musulmane ne posa jamais de problème au catholicisme. Hormis quelques procès d'hérésie fièrement revendiquée, rares, ct précoces - dans la décennie 1560 -, on peut dire, sans trop forcer la caricature, que la masse des inculpés fait de l'hérésie sans le savoir. Le tribunal inquisitorial fait oeuvre pédagogique, en réprimant les déviances rcligicuses qui, après le Concile de Trente, ne sont plus admises par les autorités ecclésiastiques, une pédagogie rude et volontairement infamante, mais rarement cruetle. Tout se joue là aussi autour de la réputation, puisque les procès inquisitoriaux sont purement idéologiques et s'appuient, en labsence de preuves matériclles, sur des témoignages à chargé et des dénonciations. Leur analyse montre d'aillcurs qu'ils s'inscrivent dans des stratégies complexes de vengeance entre individus ou groupes familiaux et que la défense de l'orthodoxie n'est bien souvent qu'un prétexte.

A des juges convaincus de la saintcté d'un serviteur de Dieu et s'efforçant de recueillir les témoignages qui vont tous dans ce sens, s'opposent donc des juges inquisitoriaux chargćs de rassembler sur le compte de l'inculpé les dénonciations destinées à établir la présomption de culpabilité. Dans ces conditions, on pourrait estimer que les cas de fausse sainteté ou de sainteté "simulée"- pour reprendre la phraséologic du temps - présents dans les archives inquisitoriales constituent le reflet inversé de la sainteté officielle, la vraie. Dans les faits, cette affirmation semble beaucoup trop simple, car la frontière est difficile à tracer. La sainteté est un 
état limite. Le comportement du saint est tapageur et contraire aux normes culturellement admisses par la société du temps. Aussi le saint franchit-il souvent cette frontière ténue entre le normal et l'anormal, au point qu'il est perçu comme un fou avant de l'être comme un saint. Dans le domaine de la mystique, il est si difficile de distingiuer le vrai du faux, l'inspiration divine de la simple simulation, que les théologiens ont mis au point une théorie élaborée du "discernement des esprits", chargée, justement d'opérer ce tri (SALLMANN, 1991). Par ailleurs, en commerçant directement avec la divinité, le saint court-circuite les pratiques habituelles du recours. Le clergé établi le perçoit alors comme un trublion, exerçant à son égard une concurrence déloyale.

C'est ainsi que plusieurs saints, dont l'authenticité fut par la suite reconnue sans conteste, furent l'objet d'enquêtes inquisitoriales. Elles furcnt généralement sans suite, mais laissèrent souvent des traces dans la réputation. La plupart du temps, l'enquête reste limitée. Elle est alors réintégrée dans le procèssus hagiographique où elle prend la forme d'un martyre. Le saint a subi avec patience la méchanceté ct les médisances de ceux qui lui voulaient du tort et, à travers lui, à Dieu lui-mêmc. Le saint en ressort grandi ct, par un effet de ricochet, le soupçon d'hérésie se transforme en vertu héroique au cours du procès de béatification. Ce rétablissement est plus difficile à opérer lorsque le procès a été mené jusqu'à son terme et a connu une grande publicité. C'est le cas de la prophétesse napolitaine Orsola Benincasa qui fut arrêtée à Rome en 1582 où elle était venue demander à Grégoire XIII la réforme de l'Eglise, jugée par un tribunal d'inquisition, libérée après avoir été reconnue non coupable, mais étroitement surveillée tout au long de sa vie. Son procès de béatification ouvert à sa mort en 1614 ne souffle mot des ennuis judiciaires de son début de carrière.

\subsection{Les cas napolitains}

Les archives inquisitoriales de Naples livrent 8 dossiers de "sainteté simulée" entre la fin du XVI ${ }^{\mathrm{e}}$ siècle et le début du XVIII" siècle, se répartissant en dcux groupes de 4 , le premier dans les années de la Réforme catholique entre 1580 et 1614 , le deuxième à la fin du XVII' siècle et au début du XVIII' siècle. Ce sont deux aspects de la sainteté qui s'opposent. Le premier groupe est formé de trois femmes, trois tertiaires, et d'un homme, un ermite. Tous ces personnages ont pour caractéristique essenticlle d'être en marge de l'institution ecclésiastique. Leur revendication de sainteré suscite immédiatement la suspicion de la part des autorités religieuses, d'autant que leur sainteté prend une totalité prophétique, messianique, apocalyptique, appelle les fidèles à la repentance et le clergê à se rêformer. Les trois tertiaires ont pour modèle Orsola Benincasa, et, audelà, la mystique médiévale à caractère prophétique, avec les figures de sainte Catherine de Sienne et de sainte Brigitte de Suède. Une seule de ces napolitaines acquit une certaine célébrité, Giulia di Marco, qui sut s'assurer des appuis auprès de l'aristocratie et du vice-roi. La papauté parvint à la discréditer, à la faire arrêter et transférer à Rome dans le plus grand secret. Elle y fut reconnue coupable d'hérésie par un tribunal d'inquisition et finit ses jours enn compagnie de ses principaux disciples dans les prisons du château Saint-Ange à Rome. Quant à l'ermite fra Ludovico, son aventure s'inspirait d'une longue tradition de l'érémitisme girovague encore bien attestée au début du $\mathrm{XVI}^{\mathrm{e}}$ siècle en Italie centrale, mais plus épisodique cn Italie méridionale. Il cut aussi un grand succès auprès des masses populaires de la ville de Naples et auprès du vice-roi séduit par son discours prophétique. Mais il est arrêté par l'inquisition en 1586 et expédié dans les geôles romaines où on perd sa trace. D'une manière générale, ces individus soupçonnés de simuler la sainteté furent sévèrement réprimés. 
II n'en va plus de même à partir de la fin du XVIle siècle, quand de nouveaux cas se présentent dans la région napolitaine. Les quatre individus poursuivis sont des hommes, deux prêtres et deux moines mendiants. La sainteté qu'ils professent n'est plus tapageuse comme celle du siècle précédent et n'a plus d'intonation politique, ils sont simplement accusés d'exercer des pouvoirs thaumaturgiques indus, soit à Naples dans les couvents féminins et dans les maisons de la bonne société citadinc, soit dans les campagnes de Naples ou de Lucanie. On perçoit bien, à travers leurs déboires, que le climat religieux s'est profondément transformé en moins d'un siècle. A une première Contre-Réforme exaltée, marquée par une attente de la "rénovation" du monde et probablement de l'arrivée des temps derniers, dans laquelle l'autorité ecclésiastique a fort à faire pour contrôler la situation, sóppose une deuxième Contre-Réforme plus assagie, plus institutionalisée où les problèmes disciplinaires l'emportent sur l'affrontement théologique. Cette constatation faite, il convient d'intcrpréter ce que les procès d'inquisition peuvent apporter à notre connaissance de la sainteté.

\subsection{L'interprétation}

11 faut avant tout dépasser les a priori de la procédure inquisitoriale ou plutôt lirc les actes des procès à la lumière du code qu'elle leur impose. Unc premièrc certitude: tous ces personnages sont vraiment des saints, car ils ont été considérés comme tel par leurs contemporains qui avaient pourtant les moyens de comparer. Ils ne sont pas, comme voudraient le faire croirc les juges, de vulgaires escrocs, des individus de mauvais aloi, voire des maniaques sexuels. La chance des historiens tient à ce que les juges inquisitoriaux sont beaucoup plus exigeants que les juges des procès de béatification. Ceux-ci se contentent d'avaliser des croyances qu'ils partagent avec les témoins, alors que ceux-là restent fondamentalement méfiants ou même franchement hostiles. Ils peuvent ainsi pousser les inculpés, lorsqu'ils les tiennent, ct les témoins dans leurs dernicrs retranchements. Les procès d'inquisition nous offrent donc une image de la sainteté bien plus nuancés et plus vivante que celle que livrent les procès de béatification

C'est lc cas en particulicr de cette sainteté mystique et messianique de la fin du XVI'ie siècle. L'autoritć de ces femmes, souvent d'hưmble extraction sociale et qui ons choisi la voie des Ticrs ordres mendiants parce qu'il leur ćtait impossible d'entrer au couvent, repose sur leurs pouvoirs mantiques. Elles sont soutenues par des petits groupes informels de dévots qui sont presque charncllement attachés à leur "Mère", dont ils attendent une communication directe avec la sphère du sacré. Certaines, comme Orsola Benincasa ou Giulia di Marco, connurent une immense popularité. Or, ces femmes étaient de grandes visionnaires et les cadres stricts des procès de béatification ne permettent pas de rccueillir ces témoignages culturcls du plus grand intérêt. Passées au crible de la censure, leurs visions perdent toute saveur, voire tout sens, et en sont réduites à de simples stérćotypes. II faut donc se reporter sur les procès d'inquisition, beaucoup plus riches en ce domaine.

Toutes ces femmes, et d'autres qui ont connu un destin semblable à travers l'Italie, on été taxées de pré-quiétistes par l'historiographie traditionnelle, comme pour mieux expliquer la rigueur de la répression. C'est évidemment absurde. Le quiétisme constitue un moment particulier de I'histoire de la spiritualité catholique et les tertiaires mystiques de Naples ont ćté pourchassées pour des raisons qu'il est facile d'apprécier. La première tient à la rivalité féroce qui sćvissait entre les ordres religieux. Une fois rentrée dans le rang, Orsola Benincasa fonda la branche féminine des Théatins qui s'efforcèrent dès lors d'écarter toute concurrence qui aurait pu nuire à 
l'image de "leur" sainte. Mais l'Eglise catholique vit aussi, aux alentours de 1600, dans l'angoisse de l'hérésie. Non pas que l'Italie du sud ait été submergée par la vague protestante. Celle-ci ne la toucha pratiquement pas et quelques procès rondement menés dans les années 1560 suffirent à dissuader tout candidat à la dissidence. Mais l'Italie fut infiltrée, d'une manière plus profonde qu'on ne le croit habituellement, par la pensée d'Erasme ou, du moins, de l'évangélisme (MENCHI, 1987). Or ces femmes, et même fra Ludovico, en exigeant la réforme de l'Eglise, en revendiquant un commerce direct avec la divinité et donc en minimisant le rôle du clergé, l'importance de certains sacrements (la confession auriculaire par exemple) et des interdits alimentaires, exprimaient de manière étouffée, peut-être même inconsciente, ce vieux rêve de l'humanisme chrétien.

En revanche, les cas de "sainteté simulée" de la fin du XVIJ" siècle et du début du XVIII siècle nous introduisent dans un tout autre climat religieux. Ils traduisent paradoxalement la grande réussite de l'Eglise contre-réformée, car ils ne possèdent aucune chargé protestataire. Ce procès ont également l'avantage d'introduire l'historien au coeur d'une sainteté moins codifiée que celle des procès de béatification, plus proche de ce qu'elle était en réalité, surtout pour ce qui constitue le noyau de la croyance, la quête thaumaturgiquc. Alors que la sainteté officielle est toujours localisée dans les villes et $s$ 'adresse donc à une tanche très restreinte de la population méridionale, certains de ces héros involontaires traduisent les aspirations du monde rural à bénćficier lui aussi des bienfaits de la sainteté. Beaucoup de témoignages viennent du monde paysan, exclu des procès de béatification. Giovanni Levi a pu étudier un cas similaire dans le Piémont de la fin du XVII' siècle (LEVI, 1985). D'autre part, ces procès d'inquisition touchent des couches du clergé à faible légitimité comme des frères lais des ordres mendiants, ou nouvelle et à l'avenir prometteur, comme les simples prêtres. Les autorités ecclésiastiques ne leur sont pas farouchement défavorables. On ne retrouve pas, dans ce documents, l'animosité que l'on peut constater dans les procès de la fin du XVI ${ }^{e}$ siècle. L'inquisition craint seulement les débordements disciplinaires et cherche à instaurer des garde-fous. L'un de ces inculpés, le frèrc Giovanni di Montesarchio, qui, en dépit de son humble statut, semble avoir joui d'un certain prestige dans les monastères franciscains de Naples, fut d'ailleurs défendu par Bonavcntura da Potenza qui bénéficiait alors d'une solide réputation de sainteté, qui lui valut d'être béatifié ai siècle suivant. Ce témoignage d'un saint sur un autre saint vaut à lui seul toutes les dépositions stéréotypées des procès de béatification.

\section{Conclusion}

Ces considérations apparaîtront peut-ĉtre bien théoriques et bien austères. Elles veulent simplement souligner à quel point l'historien reste dépendant de sources qui n'ont certainement pas été conçues dans l'intention de lui faciliter la tâche. Il les utilise, mais toujours en les détournant de leur fonction originelle. Or, pour en apprécier tout l'intérêt, il faut comprendre l'espirit de l'institution qui a présidé à leur confection et à leur mise en pratique. C'est alors seulement qu'un travail de décodage pourra s'opérer dans le but de tirer de ces documents tout ce qu'on est en droit d'en attendre, sans chercher à leur faire dire ce qu'ils ne seront jamais capables de dire. Certains m'objecteront que ce n'est là, après tout, que le b-aba de notre métier. J'en conviens, mais il m'a paru bon de le rappeler. 


\section{Bibliographie}

LEVI, Giovanni. L'eredità immaterialc. Carriera di un esorcista nel Piemonte del Scicento. Torino, Einaudi, 1985.

MARTINO, Ernesto De. La terra del rimorso. 3. ed. Milano, II Saggiatore, 1976.

Sud e magia. 4. éd. Milano, Feltrinelli, 1972.

. Mortc e pianto ritualc. Dal lamento funebre

antico al pianto di Maria. 2. éd. Rorino, Boringhieri, 1975.

MENCHI, Silvana Seidel. Erasmo in tralia, 1520-1580.

Torino, Boringhieri, 1987.
SALLMANN, Jean-Michel. Chercheurs de tresors et jeteuses de sorts. La quête du surnaturel à Naples au XVF siècle. Paris, Aubier-Montaigne, 1986.

Naples et ses saints d̀ l'âge baroque (1540. 1750). Paris, Presses Universitaires de France, 1994. (Direc.). Visions indiennes, visions baroques. Les metissages de l'inconscient. Paris, Presses Universitaires de France, 1991.

VAUCHEZ, André. La sainicté en Occident aux dernicrs siecles du Moyen Age. Rome, Ecole Française de Rome, 1981 . 